\title{
Percutaneous extraction of residual post-cholecystectomy gallstones through the T-tube tract
}

\author{
Jan Sobstyl ${ }^{D, E, F}$, Michał Sojka ${ }^{A, C, F}$, Maryla Kuczyńska ${ }^{A, B, F}$, Łukasz Światłowski ${ }^{B, C, D}$, Ewa Kuklik ${ }^{C, E, F}$, Tomasz Jargiełło ${ }^{A, B, E}$ \\ Department of Interventional Radiology and Neuroradiology, Medical University in Lublin, Poland
}

\section{Abstract}

Purpose: In the present study, the effectiveness and safety of minimally invasive percutaneous extraction of residual post-cholecystectomy gallstones through the T-tube tract were assessed.

Material and methods: Between 2000 and 2015, 12 patients (seven women and five men, mean age $78 \pm 8$ years) after open cholecystectomy with common bile duct exploration and T-tube drainage underwent percutaneous extraction of residual gallstones through the T-tube tract.

Results: The intervention was successful in $92 \%$ (11/12). In seven patients complete extraction of the retained gallstones was achieved, and in four cases partial extraction combined with passage of small residual fragments to the duodenum was obtained. In one case the extraction attempt was ineffective. Mild haemobilia was observed in two patients. No mortality or major complications were observed.

Conclusions: Our findings are consistent with literature data and confirm that percutaneous extraction of residual post-cholecystectomy gallstones through the T-tube tract is an effective and safe treatment method. Although the presented technique is not a novel approach, it can be beneficial in patients unsuitable for open surgery or laparoscopic intervention when ERCP attempt occurs ineffective or there exist contraindications to ERCP.

Key words: residual gallstones, interventional radiology, common bile duct.

\section{Introduction}

Cholelithiasis is a common condition with a prevalence of $10-20 \%$ in the general population, which increases to 24-34\% when considering patients over 70 years of age. About $30 \%$ of patients will become symptomatic, with the annual risk of symptom occurrence estimated at 1-4\% [1]. The treatment of choice for symptomatic gallstone disease is cholecystectomy, either laparoscopic or open [2]. Nowadays, approximately $95 \%$ of patients are treated with laparoscopic cholecystectomy [3]. The increased risk of conversion to open cholecystectomy is associated with a history of previous abdominal surgery, obesity, gallbladder wall thickening, acute cholecystitis, leukocytosis, or jaundice as well as suspicion of common bile duct (CBD) stones [4]. Twenty per cent of patients with gall-bladder stones have concomitant CBD stones. In these patients the management approach is cholecystectomy (open or laparoscopic) followed by observation, preoperative endoscopic retrograde cholangiopancreatography (ERCP) with subsequent cholecystectomy, intraoperative ERCP synchronous with cholecystectomy ("rendez-vous" technique), cholecystectomy with intraoperative cholangiography, and CBD exploration. Despite CBD exploration during laparoscopic or open cholecystectomy, residual CBD stones are found in $2-5 \%$ of patients [5]. Among many available treatment methods of residual CBD stones, ERCP is deemed to be the first-line man-

\footnotetext{
Correspondence address:

Jan Sobstyl, PhD, Department of Interventional Radiology and Neuroradiology, Medical University in Lublin, 8 Jaczewskiego St., 20-954 Lublin, Poland,

phone: +48 663728 258, e-mail: jan.sobstyl@gmail.com

Authors' contribution:

A Study design · B Data collection · C Statistical analysis · D Data interpretation · E Manuscript preparation · F Literature search · G Funds collection
} 
agement strategy, when ineffective laparoscopic or open surgery CBD exploration are considered. Nevertheless, in cases of poor surgical re-intervention candidates and in patients with a history of gastrointestinal surgery or anatomic anomalies that make the endoscopic intervention technically infeasible, the alternative techniques including lithotripsy and percutaneous gallstone extraction through the T-tube tract are supportive [6].

In the present study, the effectiveness and safety of minimally invasive percutaneous extraction of residual post-cholecystectomy gallstones through the T-tube tract were assessed.

\section{Material and methods}

Between 2000 and 2015, 12 patients (seven women and five men, mean age $78 \pm 8$ years) after open cholecystectomy with CBD exploration and T-tube drainage underwent percutaneous extraction of residual gallstones through the T-tube tract. The decision to refer the patients for percutaneous extraction of retained gallstones through the T-tube tract was made collectively with surgery and gastroenterology specialists. All patients were not suitable for open or laparoscopic intervention due to their general condition. Informed consent was obtained from all patients. Nine patients underwent an unsuccessful ERCP attempt, while contraindications to ERCP were present in three cases oesophageal stricture (two cases) and coagulopathy (one case). All patients were referred for the intervention six weeks after surgery. Administration of a prophylactic broad-spectrum antibiotic 30-60 min before the procedure, and continuous monitoring during the procedure, were performed in all patients. Under standard sterile conditions, T-tube cholangiography was performed to assess the location, number, and size of stones. When the stones were situated below the T-tube tip in the extrahepatic part of the $\mathrm{CBD}$, a supportive J-wire was introduced into the intrahepatic bile duct, while another 0.035 " J-wire was introduced into the CBD, close to ampulla of Vater and conversely if stones were situated in the intrahepatic bile duct. After removal of the T-tube, the introducer sheath and angulated-tip catheter were introduced over the guidewire. In cases of small stones, less than about $4 \mathrm{~mm}$ in diameter, situated below the T-tube tip in the extrahepatic part of the CBD, an initial attempt to push the stone into the duodenum with the introducer sheath was made. If the initial attempt was ineffective, thereafter through the angulated-tip catheter, a Dormia basket (Universal Stone Basket, Optimed) was introduced slightly distally to the stone, and the catheter was withdrawn. The basket was opened and retracted to engage and extract the stone through the T-tube tract (Figures 1 and 2). If traction with the Dormia basket resulted in stone fragmentation due to the stone diameter and/or impacting stones, then sizable fragments were extracted with the Dormia basket, whereas small fragments moved further through the ampulla of Vater into the duodenum (Figure 3).
Final cholangiography was performed to confirm the effective treatment outcome and total clearance of residual stones from the biliary tree. If incomplete stone extraction after fragmentation or features of haemobilia were observed, then an 8 Fr external biliary drainage catheter was inserted.

\section{Results}

In seven patients complete extraction of the retained gallstones was achieved, and in four cases partial extraction combined with passage of small residual fragments to the duodenum was obtained. In one case the extraction attempt was ineffective. Residual gallstones were situated in the intrahepatic bile duct in three cases, they were extrahepatic in seven cases, and concurrent intra- and extrahepatic in two patients. No mortality or major complications like CBD perforation, pancreatitis, or infection were observed. The patient after treatment failure was under observation, during which biliary colic with a jaundice occurred. The patient underwent open surgery stone removal. Mild haemobilia was observed in two patients. In two cases of incomplete stone extraction after fragmentation, and in two cases of mild haemobilia, an 8 Fr external biliary drainage catheter was inserted to support the appropriate decompression of the biliary tree. The catheter was removed four to seven days after the procedure, when no features of haemobilia or evidence of retained biliary stone fragments in control cholangiography were observed.

\section{Discussion}

CBD gallstones may be divided into four categories: primary - resulting from biliary accumulation in the CBD, secondary - due to displacement of already formed gallstones from the gallbladder to $\mathrm{CBD}$, residual - a result of translocation into the CBD during cholecystectomy, and recurrent stones - developing in the CBD more than three years after ductal deposit removal. Residual choledocholitiasis is a rare phenomenon, as mentioned before, affecting up to $5 \%$ of patients after cholecystectomy $[7,8]$. Predispositions to this complication are multiple, small gallstones, enlargement of the $\mathrm{CBD}$, coexistence of bile duct inflammation, pancreatitis, or presence of residual cystic duct [7].

The presenting symptoms of patients with cholelithiasis and biliary colic include sharp, intermittent, cramping right upper quadrant pain or pain radiating to the right shoulder, nausea, and vomiting. Typically, the pain occurs after a fatty meal. Fever may be present in cases of acute cholecystitis, together with jaundice, acholic stools, and dark urine when CBD stones are the cause. The mortality rate in cholecystitis is estimated at $10-30 \%$. Moreover, cholelithiasis may lead to gallstone pancreatitis and more rare complications like liver abscess, gallbladder empyema, or perforation of the gallbladder with bile peritonitis. Cholangitis, gallstone ileus, and cholecystoenteric fistula 

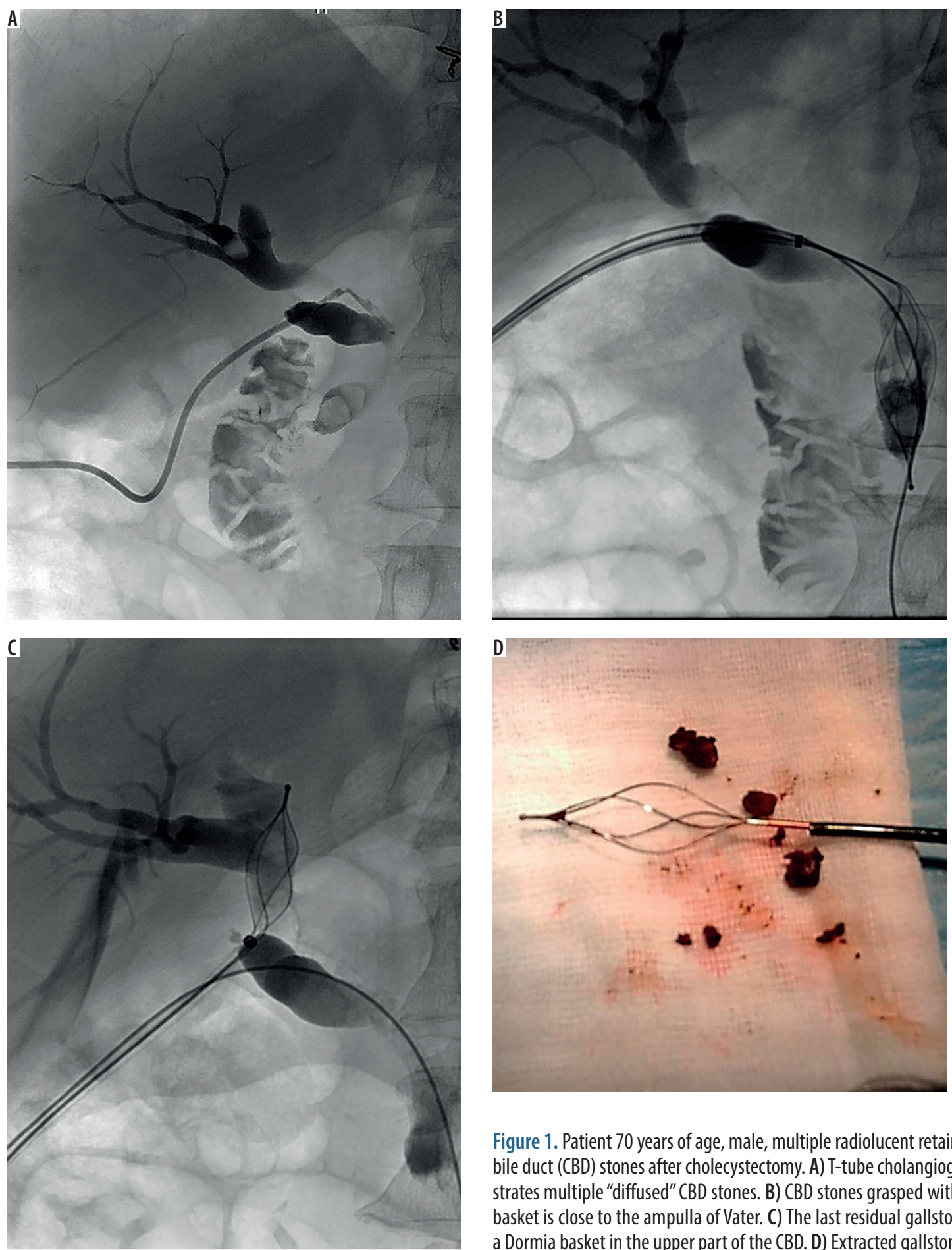

Figure 1. Patient 70 years of age, male, multiple radiolucent retained common bile duct ( $(B D)$ stones after cholecystectomy. A) T-tube cholangiogram demonstrates multiple "diffused" (BD stones. B) CBD stones grasped with the Dormia basket is close to the ampulla of Vater. C) The last residual gallstone held with a Dormia basket in the upper part of the (BD. D) Extracted gallstones

may also occur [2,9]. The coexisting factors are increased levels of alkaline phosphatase, bilirubin, liver enzymes, amylase, advanced age, and widening of the common bile duct. Transabdominal ultrasonography is considered the gold standard for the diagnosis of cholelithiasis. The sensitivity of this examination is estimated at $25-63 \%$ and its specificity at $95 \%$. The other examinations used in the diagnosis of gallstones are endoscopic ultrasonography, with sensitivity and specificity of $95 \%$ and $95-98 \%$, respectively, cholangioMR 95\% and 97\%, computed tomography $87 \%$ and $97 \%$, and cholangiography $97 \%$ and $94 \%$ [10].

The first report concerning percutaneous extraction of residual gallstones through the T-tube track was published in 1962 by Mondet [11]. In 1974, Burhenne reported results of a large study with successful extraction of residual CBD stones in 96\% (121/126) of cases. Complications occurred in $6 \%(7 / 126)$ - creation of self-limiting false passage from the sinus tract without peritoneal extravasation (two cases), septicaemia successfully treated with antibiotics (two cases), transient severe right upper quadrant pain (two cases), and vagus reflex shock (one case). No mortality was observed [12]. In 1980 Mason 

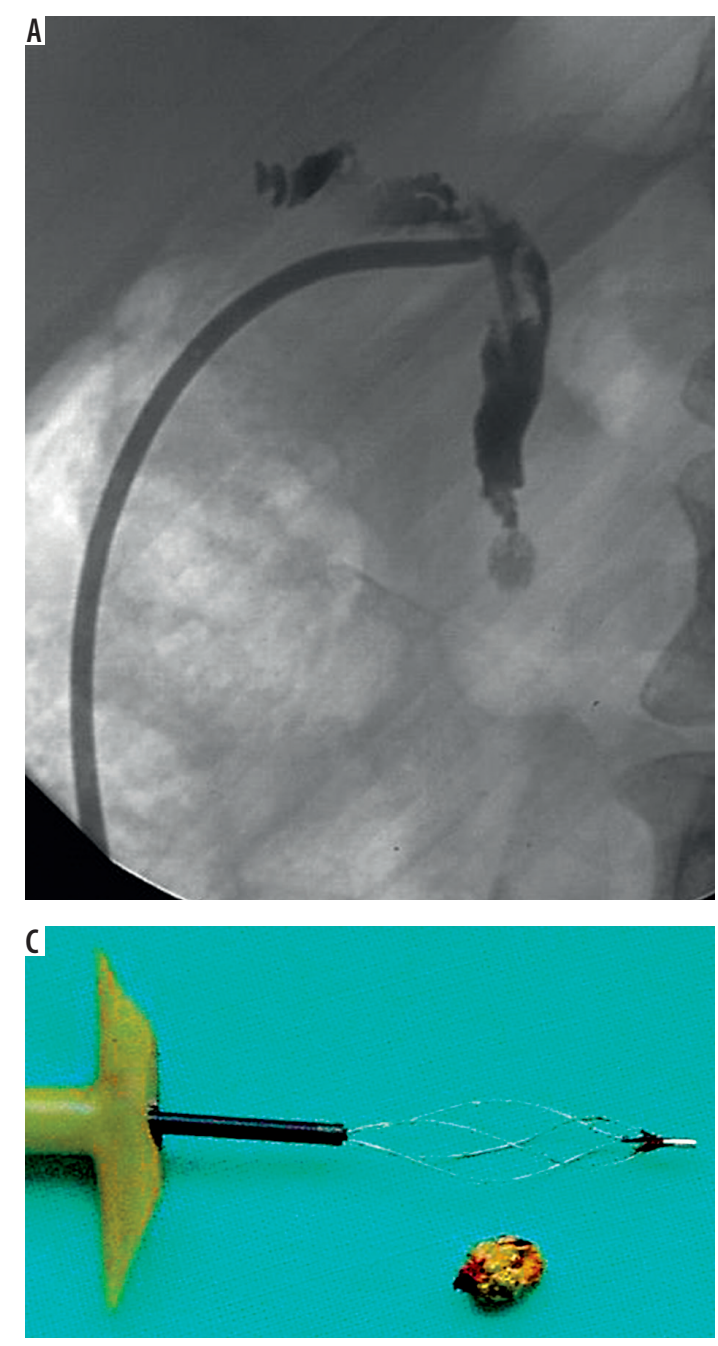

reported results obtained from 26 British institutions, with a success rate of $70 \%(92 / 131)$ and $9.2 \%(12 / 131)$ of complications - pancreatitis (one case), fever successfully treated with antibiotics (seven cases), and track perforation (four cases) with subsequent surgical treatment (one case); no mortality was observed [13]. In the subsequent years further studies demonstrated favourable outcomes of the aforementioned procedure with low risk of major complications. Brough et al. reported successful outcome in $83 \%(48 / 58)$ of cases, with only minor complications reported - one-day pyrexia (11 cases), small self-limiting bile leakage from the T-tube site (five cases), false passage without need of reintervention (one case), one-day diarrhoea and vomiting (four cases), and transient mild post-procedural discomfort with good response to analgesia (25 cases) [14]. Stokes and Clouse achieved a success rate of $93 \%$ with major complications in $8 \%$ of patients (4/53) - gallstone ileus (one case) and pancreatitis with operative stone removal (one case), and a mortality rate of $4 \%$ (2/53). Minor complications included pancreatitis (one case), small subcapsular fluid collections (two cases), small cutaneous abscess (one case), and gram-negative cholangitis (one case) that resolved with antibiotic therapy [15]. The results obtained in the reported study are as

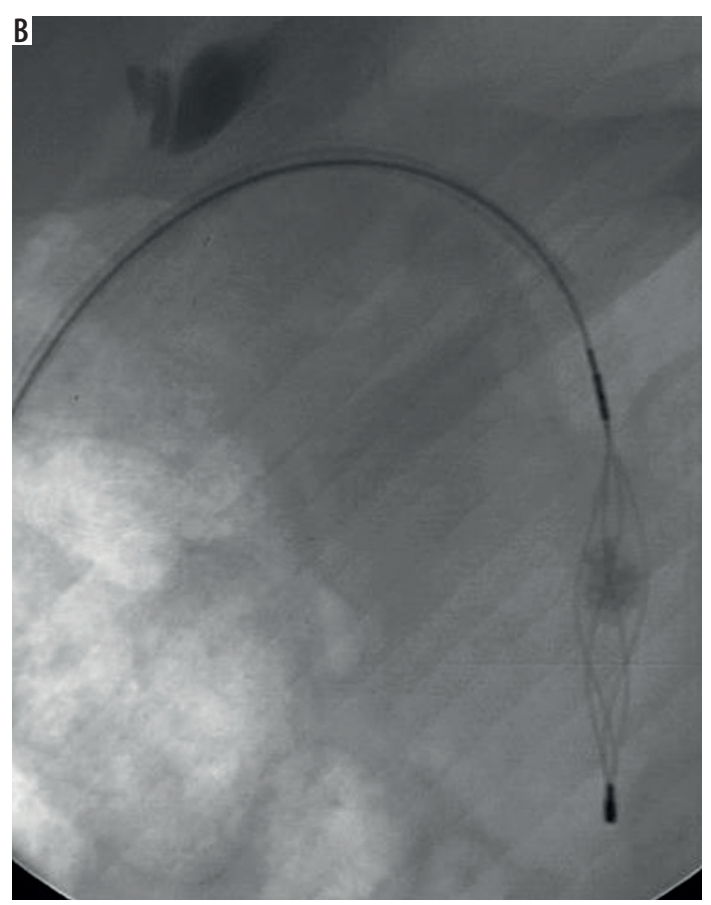

Figure 2. Patient 48 years of age, female, single radiopaque retained common bile duct (CBD) stone after cholecystectomy. A) T-tube cholangiogram demonstrates single radiopaque CBD stone close to ampulla of Vater. B) Gallstone held with a Dormia basket. C) Extracted gallstone

follows: success rate of $92 \%(11 / 12)$ and minor complications in $17 \%(2 / 12)$, with neither major complication nor mortality comparable to studies presented in the literature. The restriction of this procedure is the 4-6-week waiting period between T-tube insertion and stone extraction to allow the T-tube track to mature. Although the percutaneous treatment of the retained gallstones through the T-tube tract is usually performed with the use of specialised devices indicated for foreign body removal, such as grippers, loops, and most frequently the Dormia basket, it can also be successfully performed using a Fogarty balloon catheter. The results of such management were reported in 2016 by Ozcan et al. Percutaneous sphincteroplasty and stone expulsion into the duodenum with a Fogarty balloon catheter was performed in 89 patients with a success rate of $97.7 \%(87 / 89)$ and a $2.2 \%(2 / 89)$ complication rate intra-abdominal bile collection (one case) and distal CBD stricture (one case) $[5,6]$.

Nonetheless, endoscopic sphincterectomy is currently the major therapeutic advance in the case of residual cholelithiasis diagnosis. This procedure involves endoscopic cannulation of the bile duct and retrograde cholangiography to confirm the presence of stones. If deposits are present, Vater's sphincter is incised with an electric knife and all 

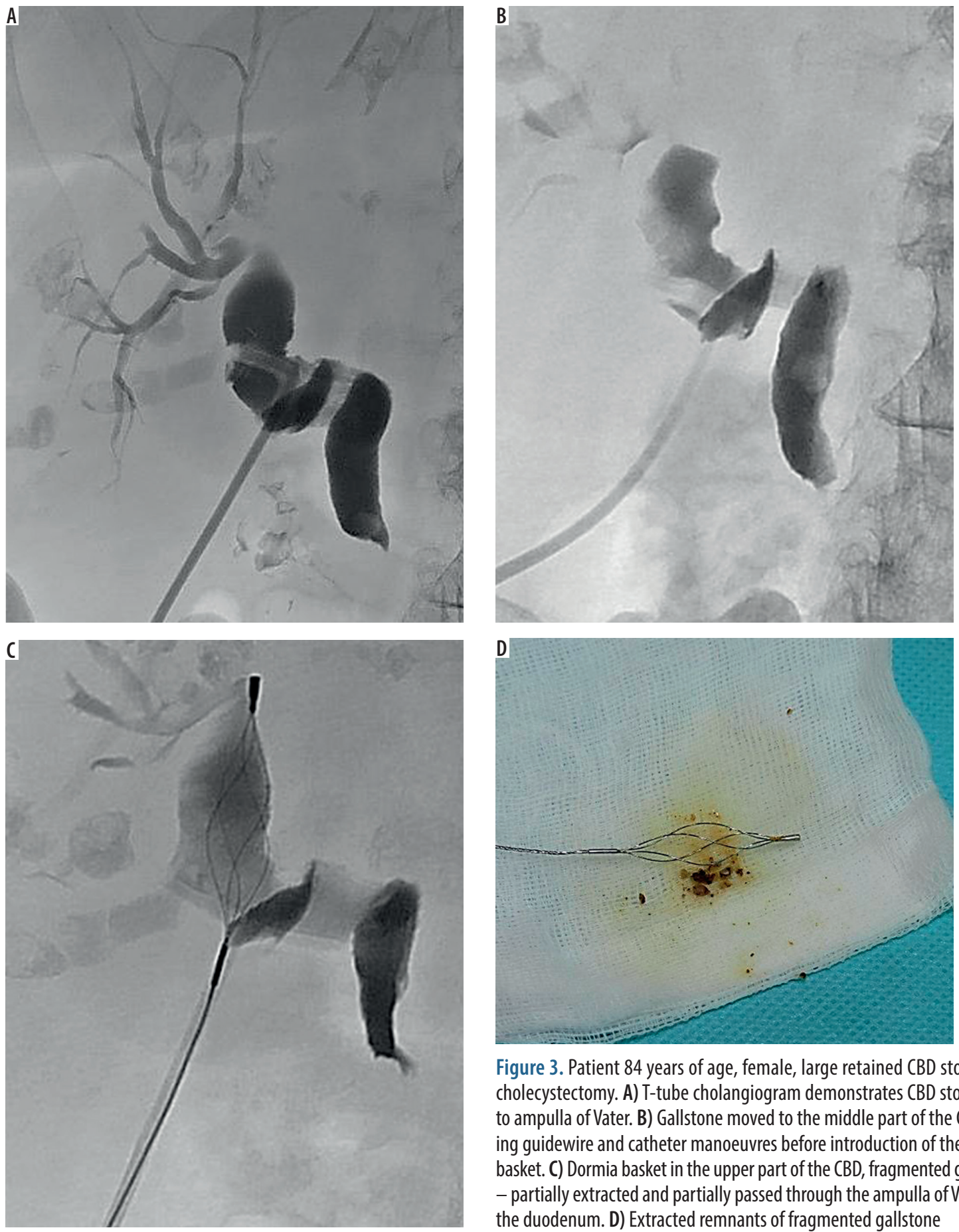

Figure 3. Patient 84 years of age, female, large retained CBD stone after cholecystectomy. A) T-tube cholangiogram demonstrates (BD stone close to ampulla of Vater. B) Gallstone moved to the middle part of the CBD during guidewire and catheter manoeuvres before introduction of the Dormia basket. C) Dormia basket in the upper part of the (BD, fragmented gallstone - partially extracted and partially passed through the ampulla of Vater into the duodenum. D) Extracted remnants of fragmented gallstone

deposits can be removed using balloon catheters - baskets (spiral, flowery, Dormia) [16]. The effectiveness of this procedure in clinical trials is $80-99 \%$ and clearly depends on the operator's experience $[17,18]$. The complications of endoscopic papillotomy include pancreatitis, cholangitis, sepsis, perforation, bleeding, and impaction of the basket; according to the literature data, their incidence is $8 \%[19,20]$. The contraindications for endoscopic papillotomy include: duodenal diverticulosis, duodenal constriction, anatomical anomalies, and advanced age of patients [7].

Different methods for residual CBD stone can be used. The most common are laparotomy or laparoscopy, both

with the similar success rates: respectively, $88.9 \%$ and $87.3 \%$. Complications that may appear are pancreatitis, sepsis, bleeding, and biliary morbidity (including bile leakage, biliary fistula, biliary peritonitis) estimated at $6.8-17.6 \%$ in the laparotomy group and $8.7 \%$ in the laparoscopy group. Total mortality is estimated at $0.14-1 \%$ after classical surgery versus $0.43 \%$ after laparoscopy. The other CBD treatment methods are lithotripsy using a holmium laser, extracorporeal lithotripsy, and electrohydraulic lithotripsy [6,19-23].

Classically, in both open and laparoscopic CBD exploration, the T-tube is inserted to prevent bile stasis, de- 
compression of the biliary tree, and lower the risk of bile leakage; the most recent studies restrict the indication for T-tube insertion to cholangitis for infected bile drainage, prevention of slender bile duct stenosis when the latter is sutured, or for patients with steadily impacted stones, to facilitate future stone extraction $[23,24]$. However, some authors consider the last indication controversial due to availability of other techniques such as cystic duct tube drainage, retrograde transhepatic biliary drainage, or internal antegrade stenting [25].

Although ERCP, laparoscopy, and open surgery are the major treatment technique of residual CBD stones, in cases were T-tube track is present alternative techniques should be considered in selected patients.

\section{Conclusions}

Our findings are consistent with literature data and confirm that percutaneous extraction of post-cholecystectomy retained gallstones through the T-tube tract is an effective and safe treatment method. Although the presented technique is not a novel approach, it can be beneficial in patients unsuitable for open surgery or laparoscopic intervention when an ERCP attempt is ineffective or there are contraindications to ERCP.

\section{Conflict of interest}

The authors report no conflict of interest.

\section{References}

1. Halldestam I, Enell EL, Kullman E, et al. Development of symptoms and complications in individuals with asymptomatic gallstones. Br J Surg 2004; 91: 734-738.

2. Duncan CB, Riall TS. Evidence-based current surgical practice: calculous gallbladder disease. J Gastrointest Surg 2012; 16: 2011 2025.

3. Williams E, Beckingham I, El Sayed G, et al. Updated guideline on the management of common bile duct stones (CBDS). Gut 2017; 66: 765-782.

4. Kama NA, Doganay M, Dolapci M, et al. Risk factors resulting in conversion of laparoscopic cholecystectomy to open surgery. Surg Endosc 2001; 15: 965-968.

5. Ozcan N, Kahriman G, Karabiyik O, et al. Percutaneous management of residual bile duct stones through T-tube tract after cholecystectomy: A retrospective analysis of 89 patients. Diagn Interv Imaging 2017; 98: 149-153.

6. Shojaiefard A, Esmaeilzadeh M, Ghafouri A, et al. Various techniques for the surgical treatment of common bile duct stones: a meta review. Gastroenterol Res Pract 2009; 2009: 840208 [cited 2018 Feb 3]. Available at: https://www.ncbi.nlm.nih.gov/pubmed/19672460.

7. Anderson WG. Choledocholithiasis after cholecystectomy. J Am Osteopath Assoc 1974; 73: 534-539.

8. Collins C, Maguire D, Ireland A, et al. A Prospective Study of Common Bile Duct Calculi in Patients Undergoing Laparoscopic Cholecystectomy: Natural History of Choledocholithiasis Revisited. Ann Surg 2004; 239: 28-33.

9. Kimura Y, Takada T, Kawarada Y, et al. Definitions, pathophysiology, and epidemiology of acute cholangitis and cholecystitis: Tokyo guidelines. J Hepatobiliary Pancreat Surg 2007; 14: 15-26.

10. Almadi MA, Barkun JS, Barkun AN. Management of suspected stones in the common bile duct. CMAJ 2012; 184: 884-892.

11. Mondet A. Tecnica de la extraction incruenta de los calculos en la litiasis residual del coledoco. Bolletin Sociedad Cirujica 1962; 46: 278.

12. Burhenne HJ. The technique of biliary duct stone extraction. Experience with 126 cases. Radiology 1974; 113: 567-572.
13. Mason R. Percutaneous extraction of retained gallstones via the T-tube track-British experience of 131 cases. Clin Radiol 1980; 31: 497-499.

14. Brough WA, Sali A, Hennessy O, et al. Percutaneous extraction of retained common bile-duct stones via the T-tube track. Aust N Z J Surg 1988; 58: 387-389.

15. Stokes KR, Clouse ME. Biliary duct stones: percutaneous transhepatic removal. Cardiovasc Intervent Radiol 1990; 13: 240-244.

16. Adler DG, Conway JD, Farraye FA, et al. Biliary and pancreatic stone extraction devices. Gastrointest Endosc 2009; 70: 603-609.

17. Wurbs D. Endoscopic papillotomy. Scand J Gastroenterol Suppl 1982; 77: 107-115.

18. Mazzeo RJ, Jordan FT, Strasius SR. Endoscopic papillotomy for recurrent common bile duct stones and papillary stenosis. A community hospital experience. Arch Surg 1983; 118: 693-695.

19. Healy K, Chamsuddin A, Spivey J, et al. Percutaneous Endoscopic Holmium Laser Lithotripsy for Management of Complicated Biliary Calculi. JSLS 2009; 13: 184-189.

20. Schreurs WH, Juttmann JR, Stuifbergen WN. Management of common bile duct stones: selective endoscopic retrograde cholangiography and endoscopic sphincterotomy: short- and long-term results. Surg Endosc 2002; 16: 1068-1072.

21. Feng Q, Huang Y, Wang K, et al. Laparoscopic Transcystic Common Bile Duct Exploration: Advantages over Laparoscopic Choledochotomy. PLoS One 2016; 11: e0162885.

22. Darkahi B, Liljeholm H, Sandblom G. Laparoscopic Common Bile Duct Exploration: 9 Years Experience from a Single Center. Front Surg 2016; 25: 23.

23. Costi R, Gnocchi A, Di Mario F, et al. Diagnosis and management of choledocholithiasis in the golden age of imaging, endoscopy and laparoscopy. World J Gastroenterol 2014; 7: 13382-13401.

24. Ambreen M, Shaikh AR, Jamal A, et al. Primary closure versus T-tube drainage after open choledochotomy. Asian J Surg 2009; 32: 21-25.

25. Yamazaki M, Yasuda H, Tsukamoto S. Primary closure of the common bile duct in open laparotomy for common bile duct stones. J Hepatobiliary Pancreat Surg 2006; 13: 398-402. 